Network Working Group

K. McKay

Request for Comments: 2658

QUALCOMM Incorporated

Category: Standards Track

August 1999

RTP Payload Format for PureVoice(tm) Audio

Status of this Memo

This document specifies an Internet standards track protocol for the Internet community, and requests discussion and suggestions for improvements. Please refer to the current edition of the "Internet Official Protocol Standards" (STD 1) for the standardization state and status of this protocol. Distribution of this memo is unlimited.

Copyright Notice

Copyright (C) The Internet Society (1999). All Rights Reserved.

\title{
ABSTRACT
}

This document describes the RTP payload format for PureVoice(tm) Audio. The packet format supports variable interleaving to reduce the effect of packet loss on audio quality.

1 Introduction

This document describes how compressed PureVoice audio as produced by the Qualcomm PureVoice CODEC [1] may be formatted for use as an RTP payload type. A method is provided to interleave the output of the compressor to reduce quality degradation due to lost packets. Furthermore, the sender may choose various interleave settings based on the importance of low end-to-end delay versus greater tolerance for lost packets.

The key words "MUST", "MUST NOT", "REQUIRED", "SHALL", "SHALL NOT", "SHOULD", "SHOULD NOT", "RECOMMENDED", "MAY", and "OPTIONAL" in this document are to be interpreted as described in RFC 2119 [3].

2 Background

The Electronic Industries Association (EIA) \& Telecommunications Industry Association (TIA) standard IS-733 [1] defines an audio compression algorithm for use in CDMA applications. In addition to being the standard CODEC for all wireless CDMA terminals, the Qualcomm PureVoice CODEC (a.k.a. Qcelp) is used in several Internet applications most notably JFax(tm), Apple(r) QuickTime(tm), and Eudora $(r)$. 
The Qcelp CODEC [1] compresses each 20 milliseconds of $8000 \mathrm{~Hz}$, 16bit sampled input speech into one of four different size output frames: Rate 1 (266 bits), Rate 1/2 (124 bits), Rate 1/4 (54 bits) or Rate 1/8 (20 bits). The CODEC chooses the output frame rate based on analysis of the input speech and the current operating mode (either normal or reduced rate). For typical speech patterns, this results in an average output of $6.8 \mathrm{k}$ bits/sec for normal mode and $4.7 \mathrm{k}$ bits/sec for reduced rate mode.

\section{RTP/Qcelp Packet Format}

The RTP timestamp is in $1 / 8000$ of a second units. The RTP payload data for the Qcelp CODEC has the following format:

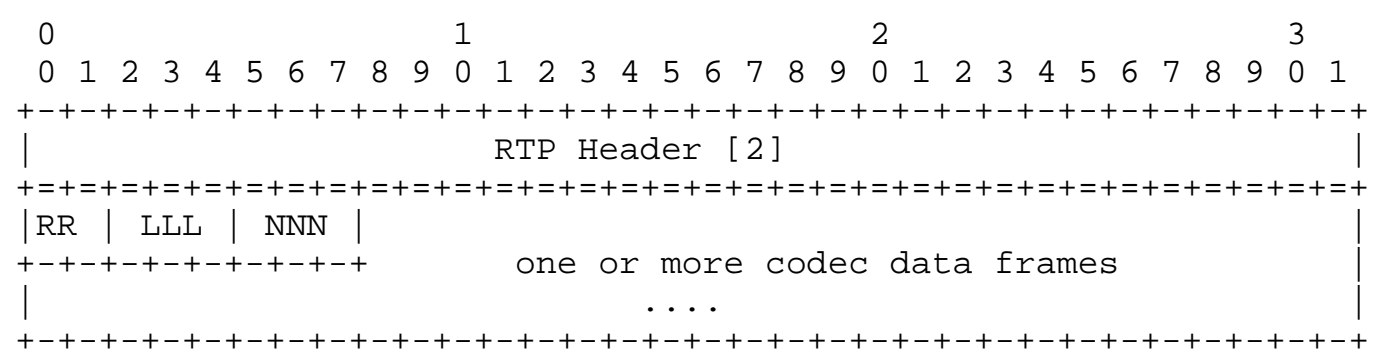

The RTP header has the expected values as described in [2]. The extension bit is not set and this payload type never sets the marker bit. The codec data frames are aligned on octet boundaries. When interleaving is in use and/or multiple codec data frames are present in a single RTP packet, the timestamp is, as always, that of the oldest data represented in the RTP packet. The other fields have the following meaning:

Reserved (RR): 2 bits MUST be set to zero by sender, SHOULD be ignored by receiver.

Interleave (LLL): 3 bits MUST have a value between 0 and 5 inclusive. The remaining two values ( 6 and 7 ) MUST not be used by senders. If this field is non-zero, interleaving is enabled. All receivers MUST support interleaving. Senders MAY support interleaving. Senders that do not support interleaving MUST set field LLL and NNN to zero.

Interleave Index (NNN): 3 bits MUST have a value less than or equal to the value of LLL. Values of NNN greater than the value of LLL are invalid. 


\subsection{Receiving Invalid Values}

On receipt of an RTP packet with an invalid value of the LLL or NNN field, the RTP packet MUST be treated as lost by the receiver for the purpose of generating erasure frames as described in section 4.

\subsection{CODEC data frame format}

The output of the Qcelp CODEC must be converted into CODEC data frames for inclusion in the RTP payload as follows:

a. Octet 0 of the CODEC data frame indicates the rate and total size of the CODEC data frame as indicated in this table:

\begin{tabular}{|c|c|c|}
\hline OCTET 0 & RATE & TOTAL CODEC data frame size (in octets) \\
\hline 0 & Blank & 1 \\
\hline 1 & $1 / 8$ & 4 \\
\hline 2 & $1 / 4$ & 8 \\
\hline 3 & $1 / 2$ & 17 \\
\hline 4 & 1 & 35 \\
\hline 5 & reserved & 8 (SHOULD be treated as a reserved value) \\
\hline $\begin{array}{l}14 \\
\text { other }\end{array}$ & $\begin{array}{l}\text { Erasure } \\
\mathrm{n} / \mathrm{a}\end{array}$ & $\begin{array}{l}1 \text { (SHOULD NOT be transmitted by sender) } \\
\text { reserved }\end{array}$ \\
\hline
\end{tabular}

Receipt of a CODEC data frame with a reserved value in octet 0 MUST be considered invalid data as described in 3.1 .

b. The bits as numbered in the standard [1] from highest to lowest are packed into octets. The highest numbered bit (265 for Rate 1, 123 for Rate 1/2, 53 for Rate 1/4 and 19 for Rate 1/8) is placed in the most significant bit (Internet bit 0 ) of octet 1 of the CODEC data frame. The second highest numbered bit (264 for Rate 1 , etc.) is placed in the second most significant bit (Internet bit 1 ) of octet 1 of the data frame. This continues so that bit 258 from the standard Rate 1 frame is placed in the least significant bit of octet 1. Bit 257 from the standard is placed in the most significant bit of octet 2 and so on until bit 0 from the standard Rate 1 frame is placed in Internet bit 1 of octet 34 of the CODEC data frame. The remaining unused bits of the last octet of the CODEC data frame MUST be set to zero. 


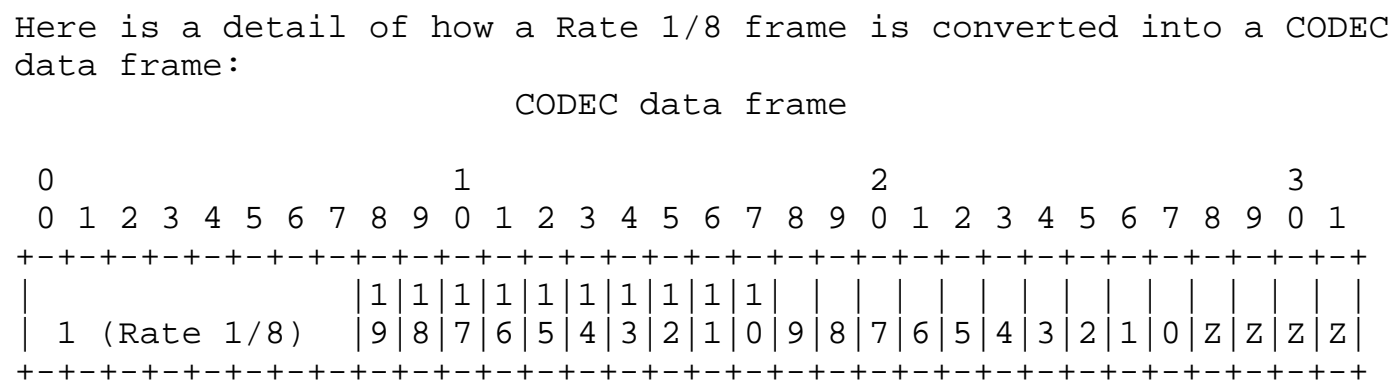

Octet 0 of the data frame has value 1 (see table above) indicating the total data frame length (including octet 0 ) is 4 octets. Bits 19 through 0 from the standard Rate $1 / 8$ frame are placed as indicated with bits marked with "Z" being set to zero. The Rate $1,1 / 4$ and $1 / 2$ standard frames are converted similarly.

\subsection{Bundling CODEC data frames}

As indicated in section 3, more than one CODEC data frame MAY be included in a single RTP packet by a sender. Receivers MUST handle bundles of up to 10 CODEC data frames in a single RTP packet.

Furthermore, senders have the following additional restrictions:

- MUST not bundle more CODEC data frames in a single RTP packet than will fit in the MTU of the RTP transport protocol. For the purpose of computing the maximum bundling value, all CODEC data frames should be assumed to have the Rate 1 size.

- MUST never bundle more than 10 CODEC data frames in a single RTP packet.

- Once beginning transmission with a given SSRC and given bundling value, MUST NOT increase the bundling value. If the bundling value needs to be increased, a new SSRC number MUST be used.

- MAY decrease the bundling value only between interleave groups (see section 3.4). If the bundling value is decreased, it MUST NOT be increased (even to the original value), although it may be decreased again at a later time. 


\subsubsection{Determining the number of bundled CODEC data frames}

Since no count is transmitted as part of the RTP payload and the CODEC data frames have differing lengths, the only way to determine how many CODEC data frames are present in the RTP packet is to examine octet 0 of each CODEC data frame in sequence until the end of the RTP packet is reached.

\subsection{Interleaving CODEC data frames}

Interleaving is meaningful only when more than one CODEC data frame is bundled into a single RTP packet.

All receivers MUST support interleaving. Senders MAY support interleaving.

Given a time-ordered sequence of output frames from the Qcelp CODEC numbered $0 \ldots n$, a bundling value $B$, and an interleave value $L$ where $n$ $=B \star(L+1)-1$, the output frames are placed into RTP packets as follows (the values of the fields LLL and NNN are indicated for each RTP packet) :

First RTP Packet in Interleave group:

$\mathrm{LLL}=\mathrm{L}, \mathrm{NNN}=0$

Frame 0, Frame L +1, Frame 2(L+1), Frame 3(L+1), ... for a total of B frames

Second RTP Packet in Interleave group: $\mathrm{LLL}=\mathrm{L}, \mathrm{NNN}=1$

Frame 1, Frame 1+L+1, Frame 1+2(L+1), Frame 1+3(L+1), ... for a total of $B$ frames

This continues to the last RTP packet in the interleave group:

L+1 RTP Packet in Interleave group:

$\mathrm{LLL}=\mathrm{L}, \mathrm{NNN}=\mathrm{L}$

Frame L, Frame L+L +1 , Frame L+2 $(\mathrm{L}+1)$, Frame L+3 $(\mathrm{L}+1), \ldots$ for a total of $B$ frames

Senders MUST transmit in timestamp-increasing order. Furthermore, within each interleave group, the RTP packets making up the interleave group MUST be transmitted in value-increasing order of the NNN field. While this does not guarantee reduced end-to-end delay on the receiving end, when packets are delivered in order by the underlying transport, delay will be reduced to the minimum possible. 
Additionally, senders have the following restrictions:

- Once beginning transmission with a given SSRC and given interleave value, MUST NOT increase the interleave value. If the interleave value needs to be increased, a new SSRC number MUST be used.

- MAY decrease the interleave value only between interleave groups. If the interleave value is decreased, it MUST NOT be increased (even to the original value), although it may be decreased again at a later time.

\subsection{Finding Interleave Group Boundaries}

Given an RTP packet with sequence number $S$, interleave value (field LLL) L, and interleave index value (field NNN) $N$, the interleave group consists of RTP packets with sequence numbers from $\mathrm{S}-\mathrm{N}$ to $\mathrm{S}-\mathrm{N}+\mathrm{L}$ inclusive. In other words, the Interleave group always consists of L+1 RTP packets with sequential sequence numbers. The bundling value for all RTP packets in an interleave group MUST be the same.

The receiver determines the expected bundling value for all RTP packets in an interleave group by the number of CODEC data frames bundled in the first RTP packet of the interleave group received. Note that this may not be the first RTP packet of the interleave group sent if packets are delivered out of order by the underlying transport.

On receipt of an RTP packet in an interleave group with other than the expected bundling value, the receiver MAY discard CODEC data frames off the end of the RTP packet or add erasure CODEC data frames to the end of the packet in order to manufacture a substitute packet with the expected bundling value. The receiver MAY instead choose to discard the whole interleave group and play silence.

\section{6 Reconstructing Interleaved Audio}

Given an RTP sequence number ordered set of RTP packets in an interleave group numbered $0 \ldots L$, where $L$ is the interleave value and $B$ is the bundling value, and CODEC data frames within each RTP packet that are numbered in order from first to last with the numbers $1 . . \mathrm{B}$, the original, time-ordered sequence of output frames from the CODEC may be reconstructed as follows:

First L+1 frames:

Frame 0 from packet 0 of interleave group

Frame 0 from packet 1 of interleave group

And so on up to...

Frame 0 from packet $L$ of interleave group 


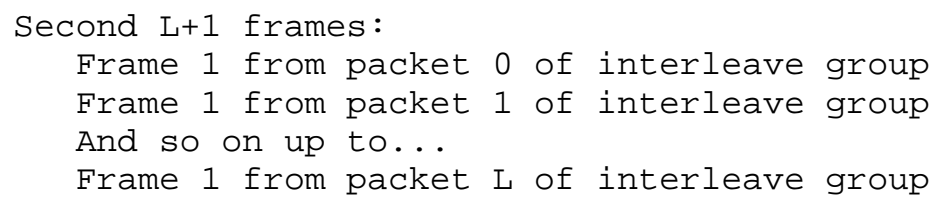

And so on up to...

Bth L+1 frames:

Frame B from packet 0 of interleave group Frame B from packet 1 of interleave group And so on up to...

Frame B from packet L of interleave group

\subsubsection{Additional Receiver Responsibility}

Assume that the receiver has begun playing frames from an interleave group. The time has come to play frame $x$ from packet $n$ of the interleave group. Further assume that packet $n$ of the interleave group has not been received. As described in section 4, an erasure frame will be sent to the Qcelp CODEC.

Now, assume that packet $\mathrm{n}$ of the interleave group arrives before frame $x+1$ of that packet is needed. Receivers SHOULD use frame $x+1$ of the newly received packet $n$ rather than substituting an erasure frame. In other words, just because packet $n$ wasn't available the first time it was needed to reconstruct the interleaved audio, the receiver SHOULD NOT assume it's not available when it's subsequently needed for interleaved audio reconstruction.

4 Handling lost RTP packets

The Qcelp CODEC supports the notion of erasure frames. These are frames that for whatever reason are not available. When reconstructing interleaved audio or playing back non-interleaved audio, erasure frames MUST be fed to the Qcelp CODEC for all of the missing packets.

Receivers MUST use the timestamp clock to determine how many CODEC data frames are missing. Each CODEC data frame advances the timestamp clock EXACTLY 160 counts.

Since the bundling value may vary (it can only decrease), the timestamp clock is the only reliable way to calculate exactly how many CODEC data frames are missing when a packet is dropped. 
Specifically when reconstructing interleaved audio, a missing RTP packet in the interleave group should be treated as containing $B$ erasure CODEC data frames where $B$ is the bundling value for that interleave group.

5 Discussion

The Qcelp CODEC interpolates the missing audio content when given an erasure frame. However, the best quality is perceived by the listener when erasure frames are not consecutive. This makes interleaving desirable as it increases audio quality when dropped packets are more likely.

On the other hand, interleaving can greatly increase the end-to-end delay. Where an interactive session is desired, an interleave (field LLL) value of 0 or 1 and a bundling factor of 4 or less is recommended.

When end-to-end delay is not a concern, a bundling value of at least 4 and an interleave (field LLL) value of 4 or 5 is recommended subject to MTU limitations.

The restrictions on senders set forth in sections 3.3 and 3.4 guarantee that after receipt of the first payload packet from the sender, the receiver can allocate a well-known amount of buffer space that will be sufficient for all future reception from the same SSRC value. Less buffer space may be required at some point in the future if the sender decreases the bundling value or interleave, but never more buffer space. This prevents the possibility of the receiver needing to allocate more buffer space (with the possible result that none is available) should the bundling value or interleave value be increased by the sender. Also, were the interleave or bundling value to increase, the receiver could be forced to pause playback while it receives the additional packets necessary for playback at an increased bundling value or increased interleave.

6 Security Considerations

RTP packets using the payload format defined in this specification are subject to the security considerations discussed in the RTP specification [2], and any appropriate profile (for example [4]). This implies that confidentiality of the media streams is achieved by encryption. Because the data compression used with this payload format is applied end-to-end, encryption may be performed after compression so there is no conflict between the two operations. 
A potential denial-of-service threat exists for data encodings using compression techniques that have non-uniform receiver-end computational load. The attacker can inject pathological datagrams into the stream which are complex to decode and cause the receiver to be overloaded. However, this encoding does not exhibit any significant non-uniformity.

As with any IP-based protocol, in some circumstances, a receiver may be overloaded simply by the receipt of too many packets, either desired or undesired. Network-layer authentication may be used to discard packets from undesired sources, but the processing cost of the authentication itself may be too high. In a multicast environment, pruning of specific sources may be implemented in future versions of IGMP [5] and in multicast routing protocols to allow a receiver to select which sources are allowed to reach it.

7 References

[1] TIA/EIA/IS-733. TR45: High Rate Speech Service option for Wideband Spread Spectrum Communications Systems. Available from Global Engineering +1 8008547179 or +1303792 2181. May also be ordered online at http://www.eia.org/eng/.

[2] Schulzrinne, H., Casner, S., Frederick, R. and V. Jacobson, "RTP: A Transport Protocol for Real-Time Applications", RFC 1889, January 1996.

[3] Bradner, S., "Key words for use in RFCs to Indicate Requirement Levels", BCP 14, RFC 2119, March 1997.

[4] Schulzrinne, H., "RTP Profile for Audio and Video Conferences with Minimal Control", RFC 1890, January 1996.

[5] Deering, S., "Host Extensions for IP Multicasting", STD 5, RFC 1112, August 1989 .

8 Author's Address

Kyle J. Mckay

QUALCOMM Incorporated

5775 Morehouse Drive

San Diego, CA 92121-1714

USA

Phone: +1 8585871121

EMail: kylemequalcomm.com 


\section{Full Copyright statement}

Copyright (C) The Internet Society (1999). All Rights Reserved.

This document and translations of it may be copied and furnished to others, and derivative works that comment on or otherwise explain it or assist in its implementation may be prepared, copied, published and distributed, in whole or in part, without restriction of any kind, provided that the above copyright notice and this paragraph are included on all such copies and derivative works. However, this document itself may not be modified in any way, such as by removing the copyright notice or references to the Internet society or other Internet organizations, except as needed for the purpose of developing Internet standards in which case the procedures for copyrights defined in the Internet Standards process must be followed, or as required to translate it into languages other than English.

The limited permissions granted above are perpetual and will not be revoked by the Internet society or its successors or assigns.

This document and the information contained herein is provided on an "AS IS" basis and THE INTERNET SOCIETY AND THE INTERNET ENGINEERING TASK FORCE DISCLAIMS ALL WARRANTIES, EXPRESS OR IMPLIED, INCLUDING BUT NOT LIMITED TO ANY WARRANTY THAT THE USE OF THE INFORMATION HEREIN WILL NOT INFRINGE ANY RIGHTS OR ANY IMPLIED WARRANTIES OF MERCHANTABILITY OR FITNESS FOR A PARTICULAR PURPOSE.

Acknowledgement

Funding for the RFC Editor function is currently provided by the Internet society. 